\title{
Post-traumatic Stress Disorder Among COVID-19 Survivors at 3-Month Follow-up After Hospital Discharge
}

\author{
Lorenzo Tarsitani, MD, $P h D^{7} \oplus$, Paolo Vassalini, $M D^{2}$, Alexia Koukopoulos, MD, PhD', \\ Cristian Borrazzo, PhD', Federica Alessi, $\mathrm{MD}^{2}$, Chiara Di Nicolantonio, $\mathrm{MD}^{\text {', }}$, \\ Riccardo Serra, $M D^{7}$, Francesco Alessandri, $M D^{3}$, Giancarlo Ceccarelli, MD, PhD, MSc², \\ Claudio Maria Mastroianni, MD, PhD' , and Gabriella d'EHtorre, MD, PhD
}

\begin{abstract}
'Department of Human Neurosciences, Policlinico Umberto I, Sapienza University of Rome, Viale dell'Università 30 , Rome, Italy; ${ }^{2}$ Department of Public Health and Infectious Diseases, Policlinico Umberto I, Sapienza University of Rome, Piazzale Aldo Moro 5, Rome, Italy; ${ }^{3}$ Intensive Care Unit, Department of General and Specialist Surgery "Paride Stefanini", Policlinico Umberto I, Sapienza University of Rome, Piazzale Aldo Moro 5, Rome, Italy.
\end{abstract}

BACKGROUND: Post-traumatic stress disorder (PTSD) is a severe but treatable mental disorder that develops after a life-threatening traumatic event. Coronavirus disease 19 (COVID-19) hospitalisation is a potentially traumatic experience, especially in severe cases. Furthermore, the unprecedented context of the severe acute respiratory syndrome coronavirus 2 pandemic, with daily media bombardment about COVID-19 mortality, may have amplified life-threatening perception also in patients with moderate infection. The aim of this study was to assess the prevalence and risk factors of PTSD at 3-month followup in patients hospitalised for COVID-19 infection.

DESIGN: In this cohort follow-up study conducted in a large Italian academic COVID-19 hospital, 115 recruited survivors were contacted by telephone 3 months after discharge to home care. The Posttraumatic Stress Disorder Checklist for DSM-5 was administered. Multivariate logistic regression models were used to analyse risk factors for the development of PTSD.

KEY RESULTS: A total of $10.4 \%$ of the sample received a PCL-5-based diagnosis of PTSD. Other 8.6\% of the sample received a diagnosis of subthreshold PTSD, which leads to significant levels of distress and impairment. Multivariate regression analysis indicated that previous psychiatric diagnosis (odds ratio $(\mathrm{OR})=6.3,95 \%$ confidence interval (CI): 3.7-78.6, $p<0.001)$ and obesity (OR = 3.51, 95\% CI: 1.4-857.9, $p=0.03$ ) were risk factors for developing PTSD. Chronic pulmonary diseases approached significance as a risk factor $(\mathrm{OR}=6.03,95 \% \mathrm{CI}: 1.0-37.1, p=0.053)$. Male sex was a protective factor $(\mathrm{OR}=0.04$, 95\% CI: 0.0 0.041, $p=0.007$ ).

CONCLUSIONS: PTSD and subthreshold PTSD rates in patients hospitalised for COVID-19 are worrying. Female sex and pre-existing mental disorders are established risk factors for PTSD, while the prospective association with obesity needs further investigation. Clinicians treating COVID-19 should consider screening for PTSD at followup assessments in patients discharged from the hospital.

Lorenzo Tarsitani and Paolo Vassalini contributed equally to this work.

Received November 10, 2020

Accepted March 16, 2021

Published online March 29, 2021
KEY WORDS: post-traumatic stress disorder; COVID-19; SARS-CoV-2; mental health; hospitalisation.

J Gen Intern Med 36(6):1702-7

DOI: $10.1007 / \mathrm{s} 11606-021-06731-7$

(C) Society of General Internal Medicine 2021

\section{INTRODUCTION}

Post-traumatic stress disorder (PTSD) is an acute, disabling mental disorder that develops after exposure to a traumatic event. Clinical manifestations include recurrent and intrusive memories, dreams or flashbacks of the trauma, avoidance of trauma-related cues, and a variety of mood and dissociative as well as cognitive symptoms. ${ }^{1}$ In the long term, PTSD is related to alcohol and substance use, mood disorders, suicidality and physical health conditions such as hypertension, obesity and coronary heart disease. ${ }^{2,3}$ The presence of clinically significant PTSD symptoms not fulfilling a full-threshold diagnosis (subthreshold or partial PTSD) has been also related to worse mental and physical health outcomes and a severe reduction in the quality of life. ${ }^{4}$ Importantly, PTSD can be effectively treated with pharmacological or psychological interventions. ${ }^{5}$

Exposure to war, physical or sexual assault, disasters and vehicle accidents are among the most common causes of PTSD. Also, acute illness and critical care admission are widely recognised as potentially traumatic events. In fact, around 1 in 5 intensive care unit survivors develops PTSD, ${ }^{6}$ with a consequent negative impact on patients' functioning, health outcomes and quality of life after the critical illness. ${ }^{7}$ One in 8 individuals admitted in emergency departments for suspected acute coronary syndrome will subsequently develop PTSD, with higher risk of mortality and cardiac event recurrence. ${ }^{8}$ Importantly, patients who rule out for acute coronary syndrome have a similar risk of PTSD compared to patients who rule in, after discharge from emergency department. ${ }^{9,10}$ It has been demonstrated that perceived life threat and fear of death play a key role in the development of PTSD, ${ }^{11}$ across events. In line with this, in cardiac acute illness subjective 
perceptions of threat during hospitalisation appear to be more strongly associated with subsequent PTSD than the final discharge diagnosis. ${ }^{10}$

Since December 2019, the novel severe acute respiratory syndrome coronavirus 2 (SARS-CoV-2), causing coronavirus disease 19 (COVID-19), has rapidly spread around the world. ${ }^{12}$ In March 2020, Italy had the second-largest number of confirmed cases, after China ${ }^{13}$. The Italian government rapidly implemented forced quarantine measures in the whole country, with a full lockdown. The government and media reported absolute figures on the daily death toll of COVID-19, in Italy and worldwide. As a result, we hypothesise that the general population perceived SARS-CoV-2 mortality to be much higher than reality. In this context, COVID-19associated hospitalisation might have been perceived as a significant life threat not only in severe cases but also in clinically stable patients as well.

To date, one study reported PTSD symptoms in $12.2 \%$ of 41 COVID-19-positive inpatients during hospitalisation. ${ }^{14}$ Two studies reported significant post-traumatic stress symptoms in $42.1 \%^{15}$ and $96.2 \%{ }^{16}$ of hospitalised COVID-19 patients. However, an early assessment of post-traumatic stress symptoms during hospitalisation may reflect temporary acute stress disorder rather than PTSD. Although peritraumatic stress symptoms are similar to post-traumatic stress symptoms, they are temporary and milder and do not usually need treatment. ${ }^{17}$

Research from past coronavirus outbreaks, especially the SARS in Hong Kong in 2003, showed PTSD symptoms and diagnosis in survivors. ${ }^{18}$

To our knowledge, no studies to date have assessed the prevalence of PTSD in COVID-19 survivors, on due time. The aim of this study was to assess the prevalence of PTSD and related risk factors at 3-month follow-up after hospital discharge to home care in patients with COVID-19 infection in a large COVID-19 university hospital in Rome, during the hot phase of the pandemic, March to April 2020. At this time, the Italian population was subject to social isolation and mandatory quarantine and was exposed to daily media reports on the raw number of deaths from COVID-19.

\section{METHODS}

\section{Subjects}

We conducted this 3-month cohort follow-up study at the Division of Infectious Diseases, Department of Public Health and Infectious Diseases, Umberto I Hospital of the Sapienza University of Rome. The Italian University Hospital Policlinico Umberto I of Rome is a 1200-bed teaching hospital, with a broad catchment area ranging between 600,000 and $1,200,000$ people. It is one of the 5 COVID-19 hospitals of Rome. ${ }^{19}$ This study is part of a larger project on health outcomes in patients affected by COVID-19 who required hospitalisation at the beginning of the pandemic. We recruited consecutive patients who were 18 years of age and older, who had been hospitalised in the Division of Infectious Diseases with confirmed COVID-19 and who were discharged to home care from March 1 to April 30. The following exclusion criteria were applied during recruitment: clinically evident cognitive impairment, active mental disorders, inadequate knowledge of the Italian language, ICU treatment in the previous 24 months. The latter was applied in order to exclude subjects with recent exposure to hospital-related traumatic episodes. Patients discharged to other wards or medical facilities were also excluded in order to avoid stress overestimation due to the inclusion of patients with non-remitting COVID-19 or unrelated clinical conditions.

All patients were approached within 1 to 2 days before discharge, to assess the eligibility for the study. They received a complete explanation of the purpose and procedures of the study and gave written informed consent to participate. Demographic and clinical variables were abstracted from medical records. In line with existing evidence, we included variables considered as potential risk factors for the development of PTSD following hospitalisation (sex, history of psychiatric diagnosis, length of hospitalisation, intensive care treatment). ${ }^{7}$ The wide-spread knowledge about comorbidity as a strong risk factor for death from COVID-19 leads us to hypothesise a heightened perception of life threat in patients with major medical comorbidities. We therefore included chronic pulmonary diseases, obesity (BMI>30) and other chronic medical illnesses in our prediction model.

Three months after discharge, patients were contacted by telephone by trained clinical raters, the information about the study and its aims were renewed, and they were asked to confirm verbally the informed consent they had provided during hospitalisation. After a detailed telephone interview in which patients were asked about their current clinical state, new medical treatment and previous diagnosis of mental disorder was confirmed. The Posttraumatic Stress Disorder Checklist for DSM-5 (PCL-5) was administered. Individuals with clinical signs of any mental disorder during the assessment were referred to the psychiatry outpatient service of our hospital or to community mental health facilities, as appropriate.

The study was approved by the ethical committee of Sapienza University of Rome (Ref. 109/2020).

\section{Instruments}

PCL-5 is a 20-item measure that assesses the presence and severity of PTSD. ${ }^{20}$ It is an updated version of the Posttraumatic Stress Disorder Checklist, ${ }^{21}$ one of the most used tools to screen PTSD worldwide, and a valid and reliable measure of PTSD. It is a 20-item 5-point Likert $(0=$ "Not at all" to 4 = "Extremely") scale assessing the 20 DSM- 5 symptoms of PTSD. A provisional PTSD diagnosis was made according to the National Center for PTSD instructions, ${ }^{20}$ considering each item rated as 2 "Moderately" or higher as a symptom endorsed, then following the DSM-5 diagnostic rule 
which requires at least 1B item (intrusion symptoms associated with the traumatic event e.g. intrusive memories, dreams, flashbacks), 1C item (avoidance of trauma-related stimuli), 2D items (cognitive and mood symptoms e.g. distorted cognitions, diminished interests, feelings of detachment) and $2 \mathrm{E}$ items (alterations in arousal and reactivity e.g. angry outbursts, self-destructive behaviour, sleep disorders). ${ }^{1}$ Patients with 3 criteria out of 4 were considered as affected by a subthreshold PTSD $^{22}$. Prevalence was also reported using total scores with a cutoff of $30 .{ }^{20}$

\section{Statistical Analysis}

All data were analysed using Statistical Package for Social Science (SPSS) version 20 or Microsoft Excel (Office 2020). Description of the median with interquartile range (IQR) 25 to $75 \%$, mean \pm standard deviation (SD), simple frequencies $(n)$, proportions (or percentages) and rates of the given data on each variable has been calculated.

A univariate analysis was used to compare patients divided into two groups: PTSD and no PTSD. The Mann-Whitney test was conducted for continuous variables and chi-square for categorical variables. A $p$-value of less than 0.05 was considered statistically significant.

In the multivariate analysis, the formula for calculating a $z$ score is $z=(x-\mu) / \sigma$, where $x$ is the raw score, $\mu$ is the population mean and $\sigma$ is the population standard deviation.

Odds ratios (ORs) and 95\% confidence intervals (95\% CIs) were calculated for all associations. Multivariate logistic regression models were used to adjust for potential confounders. $p$-value $<0.05$ was considered statistically significant.

\section{RESULTS}

Among 183 patients discharged to home care during the study period and assessed for eligibility, 68 were excluded (of them 25 did not fulfil the inclusion criteria, 46 were missed, 2 declined to participate) and 115 patients were recruited.

The recruited sample and the subjects who were lost did not differ significantly in age (median 57 (IQR 25-75\%) 48-66 vs 60.5 (IQR 25-75\%) 48-72, respectively $p=0.20$ ) and sex (male $42 \%$ vs $54 \%$ respectively $p=0.3$ ).

At the 3-month follow-up, $10.4 \%$ of the sample $(N=12)$ received a PCL-5-based diagnosis of PTSD. The other 10 patients (8.6\%) fulfilled 3 out of 4 of the DSM- 5 criteria (B C D E) at the PCL-5 and were considered as having a subthreshold diagnosis of PTSD. Twelve patients $(10.4 \%)$ had a probable diagnosis of PTSD using a PCL-5 cutoff score of 30 .

Table 1 shows demographic, clinical and treatment characteristics in all sample and in patients with or without a PCL-5based diagnosis of PTSD. A total of 26 patients (23\%) were treated with invasive or non-invasive mechanical ventilation. Among patients who developed PTSD, only 2 underwent such intensive interventions. Only 2 out of the 12 patients with PTSD were treated in ICU. Nine (8\%) patients reported a history of depressive disorder $(N=4)$, anxiety disorder $(N=$ $4)$ and unspecified mood disorder $(N=1)$.

In the multivariate logistic regression analyses, pre-existing mental disorders and obesity were found to be significant risk factors for developing PTSD. Male sex was found to be a protective factor (Table 2). Intensive care treatment was not significantly associated with the development of PTSD.

\section{DISCUSSION}

In this 3-month observational follow-up study on COVID-19 survivors discharged to home care from a large academic COVID-19 hospital, we found a provisional diagnosis of PTSD in one out of ten patients. To our knowledge, this is the first published study to report on the prevalence of PTSD with a follow-up of a least 3 months in patients hospitalised for COVID-19. Reports of PTSD symptoms during hospitalisation for COVID-19 or at discharge ${ }^{14-16}$ are hardly comparable with our results because they may reflect transitory acute stress reactions. After the outbreak of SARS in Hong Kong in 2003, Wu et al. reported a 5\% prevalence of PTSD at 3-month follow-up after discharge from the hospital, ${ }^{23}$ while in a 30-month follow-up study, it was found as high as $25.6 \%$. $^{24}$ The SARS epidemic may have led to a fear of death in infected subjects, similarly or more than the COVID-19 pandemic, due to its rapid spread and high mortality rate. ${ }^{25}$

In a recent meta-analysis, the pooled prevalence of PTSD in intensive care unit survivors at 3-month follow-up was $15.9 \%{ }^{6}$. However, ICU treatment is objectively more lifethreatening than COVID-19, with an average ICU mortality rate up to $42 \%$ in Europe. ${ }^{26}$ In our sample, $77 \%$ of patients were not in critical condition during the hospitalisation. Only two patients who subsequently developed PTSD were treated with mechanical ventilation. SARS-COV-2 infection, even without critical symptoms, represented a probable perceived life threat leading to PTSD in our patients. Our estimate of PTSD development is lower than the rates after admission in the emergency departments for suspected acute coronary syndrome which are around $15 \%$, despite discharge diagnosis. ${ }^{8-10}$ Different PTSD rates across medical conditions, however, may be due to the difference in samples, tools and times of assessment. It should be noted that our design may have underestimated the true prevalence of PTSD in COVID-19 survivors. The 3-month follow-up of our design was chosen to rapidly estimate the magnitude of PTSD rate in COVID-19 survivors, excluding acute peri-traumatic stress reactions. However, it probably underestimated the prevalence of PTSD in our sample. Indeed, although PTSD usually begins within 3 months after the traumatic event, the onset may be within 6 months ${ }^{17}$. Moreover, delayed PTSD after more than 6 months can occur in up to a quarter of cases and represents exacerbations of prior symptoms. ${ }^{27}$ Studies on ICU survivors show significant growing prevalence of PTSD according to the time 
Table 1 Demographic, Clinical and Treatment Characteristics in the Whole Sample and in PTSD Subgroups

\begin{tabular}{|c|c|c|c|c|}
\hline & All sample $(n=115)$ & PTSD $(n=12)$ & No PTSD $(n=103)$ & $\begin{array}{l}p \text {-value }{ }^{\mathrm{a}} \\
\text { PTSD vs no PTSD }^{\text {PTS }}\end{array}$ \\
\hline \multicolumn{5}{|l|}{ Sex } \\
\hline Male, $n(\%)$ & $62(54)$ & $2(17)$ & $60(58)$ & 0.007 \\
\hline Female $n(\%)$ & $53(46)$ & $10(83)$ & $43(42)$ & \\
\hline Age, median (IQR), years & $57(48-66)$ & $58(48-61)$ & $57(48-67)$ & 0.280 \\
\hline$\geq 65$ years, $n(\%)$ & $29(25)$ & $1(8)$ & $28(27)$ & 0.152 \\
\hline Length of hospital stay, median (IQR), days & $15(10-23)$ & $15(10.7-20.5)$ & $15(9.5-23.5)$ & 0.426 \\
\hline Intensive care treatment, $n(\%)$ & $26(23)$ & $2(17)$ & $24(23)$ & 0.638 \\
\hline Previous psychiatric diagnosis, $n(\%)$ & $9(8)$ & $5(42)$ & $4(4)$ & $<0.001$ \\
\hline Obesity (BMI>30), $n(\%)$ & $5(4)$ & $2(17)$ & $3(3)$ & 0.027 \\
\hline Chronic pulmonary diseases ${ }^{\mathrm{b}}, n(\%)$ & $15(13)$ & $4(33)$ & $11(11)$ & 0.034 \\
\hline Other chronic medical illness ${ }^{\mathrm{c}}$ & $60(52)$ & $5(42)$ & $55(53)$ & 0.472 \\
\hline
\end{tabular}

Abbreviations: PTSD, post-traumatic stress disorder; BMI, body mass index

${ }^{a}$ Comparison between patients with PTSD and those without PTSD

${ }^{b}$ Include Chronic Obstructive Pulmonary Disease and Asthma

${ }^{c}$ Include cerebrovascular disease, inflammatory bowel diseases, hypothyroidism, diabetes, hypertension, history or present neoplasm, coronary heart disease

point of assessment, from 3 to 12 months. ${ }^{6}$ Accordingly, patients in our sample, especially those with partial PTSD, may develop a full-criteria PTSD after the assessment. Future research is needed to estimate the prevalence of PTSD at longer follow-up. Another possible source of underestimation of the prevalence of PTSD in our study is the exclusion of patients discharged to other wards or medical facilities. Individuals with more severe or non-remitting COVID-19 or with serious comorbidities could have been exposed to higher threat perception as compared to patients discharged to home.

The $8.6 \%$ of our sample was considered as having a subthreshold diagnosis of PTSD and can be considered at risk of worse health outcome. ${ }^{4}$ This result was expected while prevalence rates of subthreshold PTSD are similar to those of full PTSD across studies. ${ }^{4}$ Research has consistently shown that people with subthreshold PTSD have significant levels of distress and impairment. ${ }^{28}$ Individuals with subthreshold PTSD are also at risk of depressive symptoms, suicidal ideation, alcohol use and other comorbidities. ${ }^{4}$

Male sex was a significant protective factor against PTSD in our sample, which is supported by some previous studies in ICU survivors. ${ }^{7}$ In general, women have a higher risk of developing PTSD as compared to men. ${ }^{29}$

Pre-existing psychiatric diagnosis (history of anxiety and depressive disorders, in our sample) was the strongest risk factor for PTSD in our analysis. A history of psychiatric disorders,

Table 2 Multivariate Logistic Regression Model Predicting PTSD

\begin{tabular}{llc}
\hline \hline Variables & OR (95\% CI) & $p$-value \\
\hline Sex, male & $\mathbf{0 . 0 4}(\mathbf{0 . 0}-\mathbf{0 . 0 4 1})$ & $\mathbf{0 . 0 0 7}$ \\
Age $(\geq 65)$ & $1.03(0.9-1.1)$ & 0.270 \\
Length of hospital stay $(\geq 15)$ & $1.01(0.9-1.1)$ & 0.797 \\
Intensive care treatment & $1.75(0.2-18.8)$ & 0.646 \\
Obesity (BMI>30) & $\mathbf{3 . 5 1}(\mathbf{1 . 4 - 8 5 7 . 9 )}$ & $\mathbf{0 . 0 3 1}$ \\
Previous psychiatric diagnosis & $\mathbf{6 . 3 0}(\mathbf{3 . 7 - 7 8 . 6 )}$ & $\mathbf{< 0 . 0 0 1}$ \\
Chronic pulmonary diseases & $6.03(1.0-37.1)$ & 0.053 \\
Other chronic medical illness & $3.08(0.2-38.1)$ & 0.381 \\
\hline
\end{tabular}

Bold font indicates statistical significance especially anxiety and depressive disorders, was found as a risk factor for PTSD in ICU survivors. ${ }^{7}$ This finding is also in congruence with previous research on PTSD following natural and human-made disasters as well as other common traumas. ${ }^{28,}$ 30

Obesity was a significant predictor of PTSD in our sample. While PTSD and history of trauma are recognised as risk factors for obesity, ${ }^{31}$ the reverse relationship was never reported. Inconsistent evidence exists regarding a possible bidirectional relationship between obesity and other mental disorders, especially depressive disorders. ${ }^{32}$ Chronic pulmonary disease was bivariately associated with PTSD (Table 1); however, in the multivariate analysis, it only approached significance ( $p=$ 0.053). These results are in line with emerging evidence showing that obesity and chronic pulmonary disease leads to higher risk of developing serious events and worse outcome in COVID-19. ${ }^{33,} 34$ Therefore, life threat perception was probably higher in patients with severe COVID-19 infection. Additionally, during the first phase of the COVID-19 pandemic, chronic pulmonary disease and obesity were consistently reported by media as risk factors for SARS-CoV-2 mortality. We hypothesise that people with obesity and chronic pulmonary disease hospitalised for COVID-19 infection had a higher perception of threat compared to healthy individuals, despite clinical presentation. Future studies are needed to assess perceived life threat and their determinants in patients hospitalised for COVID-19.

This study has to be interpreted in the light of several limitations. The small sample size, the single-centre design of our study and the unprecedented context of the first phase of the COVID-19 pandemic may have greatly limited the generalisability to other settings. Furthermore, although our multivariable logistic regression approach resulted statistically significant, the sample size is smaller than the one required for a reliable multivariate regression analysis. Additionally, possible collinearities of chronic pulmonary diseases and other chronic medical illnesses were not investigated. As a 
consequence, findings on PTSD risk factors should be accepted with caution and need to be replicated by future studies on larger samples. Another limitation is the use of medical records to collect clinical variables and the telephone interview methodology. Although unfeasible during the COVID-19 pandemic emergency, a face-to-face assessment of clinical baseline variables and a structured psychiatric interview to diagnose PTSD at follow-up would have been preferable. Moreover, the lack of assessment of life threat during hospitalisation is a major limitation of our study. Future research is warranted to verify if media reports of absolutes figures on lethal cases of COVID-19 may distort life threat perceptions. Furthermore, 46 patients were lost to follow-up. Although age and sex were similar between missing subjects and those evaluated, this does not rule out the possibility of bias.

In conclusion, one out of five patients hospitalised for COVID-19 was diagnosed with PTSD or subthreshold PTSD at 3-month follow-up. Female sex and previous mental disorders were significant risk factors. Obesity predicted the development of PTSD and reason for this unexpected association should be further investigated.

PTSD is a serious but treatable condition. Clinicians treating COVID-19 in hospitals should be aware of PTSD risk. When feasible, screening and intervention might reduce the impact of PTSD in COVID-19 survivors.

Corresponding Author: Lorenzo Tarsitani, MD, PhD; Department of Human Neurosciences, Policlinico Umberto I, Sapienza University of Rome, Viale dell'Università 30, 00185Rome, Italy (e-mail: lorenzo. tarsitani@uniroma1.it).

\section{Declarations:}

Conflict of Interest: The authors declare that they do not have a conflict of interest.

\section{REFERENCES}

1. American Psychiatric Association. American Psychiatric Association: Diagnostic and Statistical Manual of Mental Disorders Fifth Edition.; 2013.

2. McFarlane AC. The long-term costs of traumatic stress: Intertwined physical and psychological consequences. World Psychiatry 2010;9(1):310. doi:https://doi.org/10.1002/j.2051-5545.2010.tb00254.x

3. Pacella ML, Hruska B, Delahanty DL. The physical health consequences of PTSD and PTSD symptoms: A meta-analytic review. J Anxiety Disord 2013;27(1):33-46. doi:https://doi.org/10.1016/j.janxdis.2012.08.004

4. Bergman HE, Kline AC, Feeny NC, Zoellner LA. Examining PTSD Treatment Choice Among Individuals with Subthreshold PTSD. Behav Res Ther 2015;73:33. doi:https://doi.org/10.1016/J.BRAT.2015.07.010

5. Bryant RA. Post-traumatic stress disorder: a state-of-the-art review of evidence and challenges. World Psychiatry 2019;18(3):259-269. doi:https://doi.org/10.1002/wps.20656

6. Righy C, Rosa RG, Da Silva RTA, et al. Prevalence of post-traumatic stress disorder symptoms in adult critical care survivors: A systematic review and meta-analysis. Crit Care 2019;23(1):1-13. doi:https://doi.org/10. 1186/s13054-019-2489-3

7. Davydow DS, Gifford JM, Desai S V., Needham DM, Bienvenu OJ. Posttraumatic stress disorder in general intensive care unit survivors: a systematic review. Gen Hosp Psychiatry 2008;30(5):421-434. doi:https://doi.org/10.1016/j.genhosppsych.2008.05.006
8. Edmondson D, Kronish IM, Shaffer JA, Falzon L, Burg MM. Posttraumatic stress disorder and risk for coronary heart disease: A meta-analytic review. Am Heart J 2013;166(5):806-814. doi:https://doi.org/10.1016/j. ahj.2013.07.031

9. Kronish IM, Edmondson D, Moise N, et al. Posttraumatic stress disorder in patients who rule out versus rule in for acute coronary syndrome. Gen Hosp Psychiatry 2018;53:101-107. doi:https://doi.org/10.1016/j. genhosppsych.2018.02.007

10. Meli L, Birk J, Edmondson D, Bonanno GA. Trajectories of posttraumatic stress in patients with confirmed and rule-out acute coronary syndrome. Gen Hosp Psychiatry 2020;62:37-42. doi:https://doi.org/10.1016/j. genhosppsych.2019.11.006

11. Heir T, Blix I, Knatten CK. Thinking that one's life was in danger: Perceived life threat in individuals directly or indirectly exposed to terror. Br J Psychiatry 2016;209(4):306-310. doi:https://doi.org/10.1192/bjp. bp. 115.170167

12. Zhu N, Zhang D, Wang W, et al. A novel coronavirus from patients with pneumonia in China, 2019. N Engl J Med 2020;382(8):727-733. doi:https://doi.org/10.1056/NEJMoa2001017

13. Remuzzi A, Remuzzi G. COVID-19 and Italy: what next? Lancet. 2020;395(10231):1225-1228. doi:https://doi.org/10.1016/S01406736(20)30627-9

14. Qi R, Chen W, Liu S, et al. Psychological morbidities and fatigue in patients with confirmed COVID-19 during disease outbreak: prevalence and associated biopsychosocial risk factors. medRxiv Prepr Serv Heal Sci. Published online 2020. doi:https://doi.org/10.1101/2020.05.08. 20031666

15. Wesemann U, Hadjamu N, Willmund G, et al. Influence of COVID-19 on general stress and posttraumatic stress symptoms among hospitalized high-risk patients. Psychol Med 2020;2:1-2. doi:https://doi.org/10. 1017/s0033291720003165

16. Bo HX, Li W, Yang Y, et al. Posttraumatic stress symptoms and attitude toward crisis mental health services among clinically stable patients with COVID-19 in China. Psychol Med. 2020:1. doi:https://doi.org/10.1017/ S0033291720000999

17. APA - Diagnostic and Statistical Manual of Mental Disorders DSM-5 Fifth Edition. Accessed September 25, 2020. https://www.appi.org/Diagnostic_and_Statistical_Manual_of_Mental_Disorders_DSM-5_Fifth_Edition

18. Kaseda ET, Levine AJ. Post-traumatic stress disorder: a differential diagnostic consideration for COVID-19 survivors. Published online 2020. doi:https://doi.org/10.1080/13854046.2020.1811894

19. Ceccarelli G, Spagnolello O, Borrazzo C, et al. Impact of the 2017 measles outbreak on the emergency care system of a large tertiary-care teaching hospital in Italy: A retrospective analysis. Eur $\mathrm{J}$ Pub Health 2019;29(5):966-971. doi:https://doi.org/10.1093/eurpub/ckz056

20. Weathers FW, Litz BT, Keane TM, Palmieri PA, Marx BP, Schnurr PP. The PTSD Checklist for DSM-5 (PCL-5). Natl Cent PTSD. Published online 2013. doi:https://doi.org/10.1037/t02622-000

21. Blanchard EB, Jones-Alexander J, Buckley TC, Forneris CA. Psychometric properties of the PTSD checklist (PCL). Behav Res Ther 1996;34(8):669-673. doi:https://doi.org/10.1016/0005-7967(96)00033-2

22. Mclaughlin KA, Koenen KC, Friedman MJ, et al. Subthreshold posttraumatic stress disorder in the world health organization world mental health surveys. Biol Psychiatry 2015;77(4):375-384. doi:https://doi.org/ 10.1016/j.biopsych.2014.03.028

23. Wu KK, Chan SK, Ma TM. Posttraumatic stress after SARS. Emerg Infect Dis 2005;11(8):1297-1300. doi:https://doi.org/10.3201/eid1108. 041083

24. Mak IWC, Chu CM, Pan PC, Yiu MGC, Chan VL. Long-term psychiatric morbidities among SARS survivors. Gen Hosp Psychiatry 2009;31(4):318-326. doi:https://doi.org/10.1016/j.genhosppsych.2009. 03.001

25. Wu P, Fang Y, Guan $Z$, et al. The psychological impact of the SARS epidemic on hospital employees in China: Exposure, risk perception, and altruistic acceptance of risk. Can J Psychiatr 2009;54(5):302-311. doi:https://doi.org/10.1177/070674370905400504

26. Weigl W, Adamski J, Goryński P, Kański A, Hultström M. ICU mortality and variables associated with ICU survival in Poland. Eur $\mathrm{J}$ Anaesthesiol 2018;35(12):949-954. doi:https://doi.org/10.1097/EJA. 0000000000000889

27. Smid GE, Mooren TTM, Van Der Mast RC, Gersons BPR, Kleber RJ. Delayed posttraumatic stress disorder: Systematic review, meta-analysis, and meta-regression analysis of prospective studies. J Clin Psychiatr 2009;70(11): 1572-1582. doi:https://doi.org/10.4088/JCP.08r04484 
28. Sareen J. Posttraumatic stress disorder in adults: Impact, comorbidity, risk factors, and treatment. Can J Psychiatr 2014;59(9):460-467. doi:https://doi.org/10.1177/070674371405900902

29. Olff M. Sex and gender differences in post-traumatic stress disorder: an update. Eur J Psychotraumatol. 2017;8(sup4):1351204. doi:https://doi. org/10.1080/20008198.2017.1351204

30. Bromet EJ, Atwoli L, Kawakami N, et al. Post-traumatic stress disorder associated with natural and human-made disasters in the World Mental Health Surveys. Psychol Med 2017;47(2):227-241. doi:https://doi.org/ $10.1017 /$ S0033291716002026

31. Suliman S, Anthonissen L, Carr J, et al. Posttraumatic stress disorder, overweight, and obesity: A systematic review and meta-analysis. Harv Rev Psychiatr 2016;24(4):271-293. doi:https://doi.org/10.1097/HRP. 0000000000000106
32. Rajan TM, Menon V. Psychiatric disorders and obesity: A review of association studies. J Postgrad Med 2017;63(3):182-190. doi:https://doi. org/10.4103/jpgm.JPGM_712_16

33. Tartof SY, Qian L, Hong V, et al. Obesity and Mortality Among Patients Diagnosed With COVID-19: Results From an Integrated Health Care Organization. Ann Intern Med 2020;173(10):773-781. doi:https://doi. org/10.7326/M20-3742

34. Nandy K, Salunke A, Pathak SK, et al. Coronavirus disease (COVID-19): A systematic review and meta-analysis to evaluate the impact of various comorbidities on serious events. Diabetes Metab Syndr Clin Res Rev 2020; 14(5):1017-1025. doi:https://doi.org/10.1016/j.dsx.2020.06.064

Publisher's Note: Springer Nature remains neutral with regard to jurisdictional claims in published maps and institutional affiliations. 